\title{
Short-range charge density wave order in $\mathrm{La}_{1.88} \mathrm{Sr}_{0.12} \mathrm{CuO}_{4}$ under uniaxial pressure
}

\author{
R. Frison ${ }^{1}$, J. Küspert ${ }^{1}$, Q. Wang ${ }^{1}$, N.B.C. Christensen ${ }^{2}$, J. Choi ${ }^{3}$, O. Ivashko ${ }^{4}$, M. v. Zimmermann ${ }^{4}$, T. Kurosawa ${ }^{5}$, N. \\ Momono $^{6}, \mathrm{M}^{2} \mathrm{Oda}^{5}$ and $\mathrm{J} \mathrm{Chang}^{1}$ \\ ${ }^{I}$ Physik Institut, University of Zurich, Winterthurerstrasse 190, CU-8057 Zurich; \\ ${ }^{2}$ Department of Physics, Technical University of Denmark, DK-2800 Kongens Lyngby, Denmark; \\ ${ }^{3}$ Diamond Light Source, Harwell Science \& Innovation Campus Didcot Oxfordshire OX11 0DE,United Kingdom; \\ ${ }^{4}$ Deutsches Elektronen-Synchrotron DESY, Notkestraße 85, 22607 Hamburg, Germany; \\ ${ }^{5}$ Department of Physics, Hokkaido University - Sapporo 060-0810, Japan; \\ ${ }^{6}$ Department of Applied Sciences, Muroran Institute of Technology, Muroran 050-8585, Japan.
}

ruggero.frison@physik.uzh.ch

In cuprate materials, copper-oxide based perovskites, high-temperature superconductivity microscopically intertwines [1] with the pseudogap phase [2,3], charge-density-wave (CDW) order [4-5], as well as electronic nematic phases [6]. The mechanisms underlying the emergence of superconductivity, the nature of the pseudogap phase and the symmetry properties of the density-wave states remain to be clarified.

In the $\mathrm{La}-\mathrm{Sr}-\mathrm{Cu}-\mathrm{O}$ system the ubiquitous presence of twin domains [7] prevents to unambiguously establish the true nature of CDW order. Under these conditions, in fact, the diffraction signatures of a uniaxial stripe CDW order [4] are indistinguishable from those of biaxial structures in which the charge density is simultaneously modulated along two perpendicular directions.

Here we report on our high-energy $(100 \mathrm{keV}) \mathrm{X}$-ray diffraction experiments carried at the P21.1 beamline at PETRA III (DESY) showing that applying uniaxial pressure to $\mathrm{La}_{1.88} \mathrm{Sr}_{0.12} \mathrm{CuO}_{4}$ (LSCO) it is possible to resolve the domain degeneracy and thereby uncover the underlying charge stripe structure. We find that the resulting charge stripe ordering vector is perpendicular to the uniaxial stress direction. We discuss the average symmetry transition from the high temperature tetragonal (HTT) to the low-temperature orthorhombic (LTO) showing signatures of a possible breaking of the lattice centering and its link to the symmetry of the CDW order. Using a first-of-its kind dataset of CDW peaks, collected with a 2D single-photon counting detector, we attempt to resolve the underlying structure modulation in terms of in-plane and out-of-plane ionic displacements and discuss our finding within the bounding limits imposed by the evidence of an extended stacking-type disorder along the $c$-axis.

[1] Fradkin E., Kivelson S. A., Tranquada J. M., (2015) Rev. Mod. Phys. 87, 457-482.

[2] Norman M. R., Pines D., Kallin C., (2005) Advances in Physics 54, 715-733.

[3] Daou R., Chang J., LeBoeuf D., Cyr-Choinière O., Laliberté F., Doiron-Leyraud N., Ramshaw B. J., Liang R., Bonn D. A., Hardy W. N., Taillefer L., (2010) Nature 463, 519-522.

[4] Tranquada J. M., Sternlieb B. J., Axe J. D., Nakamura Y., Uchida S., (1995), Nature 375, 561-563.

[5] Wu T., Mayaffre H., Krämer S., Horvatic M., Berthier C., Hardy W. N., Liang R., Bonn D. A., Julien M., (2011), Nature 477, 19.

[6] Murayama H., Sato Y., Kurihara R., Kasahara S., Mizukami Y., Kasahara Y., Uchiyama H., Yamamoto A., Moon E. G., Cai J., Freyermuth J., Greven M., Shibauchi T., Matsuda Y., (2019) Nature Communications 10, 3282.

[7] Braden M., Heger G., Schweiss P., Fisk Z., Gamayunov K., Tanaka I., Kojima H., (1992) Physica C $191,455$.

Keywords: Charge-order; Phase transitions; Diffuse scattering 